\title{
Effects of gaze on vection from jittering, oscillating, and purely radial optic flow
}

\author{
Stephen Palmisano and Juno Kim \\ University of Wollongong, Wollongong, New South Wales, Australia
}

\begin{abstract}
In this study, we examined the effects of different gaze types (stationary fixation, directed looking, or gaze shifting) and gaze eccentricities (central or peripheral) on the vection induced by jittering, oscillating, and purely radial optic flow. Contrary to proposals of eccentricity independence for vection (e.g., Post, 1988), we found that peripheral directed looking improved vection and peripheral stationary fixation impaired vection induced by purely radial flow (relative to central gaze). Adding simulated horizontal or vertical viewpoint oscillation to radial flow always improved vection, irrespective of whether instructions were to fixate, or look at, the center or periphery of the self-motion display. However, adding simulated high-frequency horizontal or vertical viewpoint jitter was found to increase vection only when central gaze was maintained. In a second experiment, we showed that alternating gaze between the center and periphery of the display also improved vection (relative to stable central gaze), with greater benefits observed for purely radial flow than for horizontally or vertically oscillating radial flow. These results suggest that retinal slip plays an important role in determining the time course and strength of vection. We conclude that how and where one looks in a self-motion display can significantly alter vection by changing the degree of retinal slip.
\end{abstract}

Although multiple senses contribute to the perception of self-motion, visual and vestibular information appear to dominate this experience (Dichgans \& Brandt, 1978; Gibson, 1966; Howard, 1982). Vision is able to detect both constant and accelerating self-motions from the optic flow presented to our moving eyes (Berthoz, Pavard, \& Young, 1975; Dichgans \& Brandt, 1978; Johansson, 1977; Lishman \& Lee, 1973). By contrast, the vestibular system of the inner ear is able only to detect self-accelerations, on the basis of the inertia of the fluid in the semicircular canals and otolith organs (Dichgans \& Brandt, 1978). Most explanations of how these two senses interact in the perception of self-motion are based on the notion of sensory conflict (see Oman, 1982; Reason, 1978; Reason \& Brand, 1975; Zacharias \& Young, 1981). For example, it has long been known that there can be substantial delays in the onset of visually induced illusions of self-motion (known as vection; Melcher \& Henn, 1981; Teixeira \& Lackner, 1979; Wong \& Frost, 1981; Young, Dichgans, Murphy, \& Brandt, 1973; Zacharias \& Young, 1981). Sensory conflict theories propose that such delays occur because salient visual-vestibular conflicts are generated when stationary observers are first exposed to a visual self-motion display, since they expect to be accelerated up to the speed represented by this display, but the vestibular stimulation that normally accompanies such a self-acceleration is absent (e.g., Zacharias \& Young, 1981). These theories predict that the initially salient visual-vestibular conflict will fade rapidly when the optic flow simulates constant velocity self-motion (because little or no vestibular input is expected in this situation) and that, after a short time, compelling vection will be induced. If, on the other hand, the optic flow simulates large and frequent changes to the speed and direction of self-motion, it is predicted that salient visual-vestibular conflicts should persist and prevent the induction of compelling vection.

Sensory conflict accounts of self-motion perception have recently been challenged by findings that compelling vection can be induced in situations thought to generate significant and sustained visual-vestibular conflicts (Palmisano, Burke, \& Allison, 2003; Palmisano, Gillam, $\&$ Blackburn, 2000). The jittering and nonjittering displays used in these studies all contained the same radial flow component, which simulated constant-velocity forward self-motion in depth, and were thus expected to generate only transient visual-vestibular conflict in stationary observers. However, jittering displays also contained an additional flow component that simulated random horizontal and/or vertical changes to the observer's viewpoint. The large, frequent head movements simulated by these jittering radial flow displays (up to $30 \mathrm{~Hz}$ ) were expected to produce significant and sustained visual-vestibular conflicts in stationary observers. Contrary to the notion that increased visual-vestibular conflict always impairs vection, jittering radial flow was found to induce experiences of vection in depth that started sooner, lasted longer, and were more compelling than those induced by nonjittering radial flow. Subsequent research has shown that simulated 
viewpoint oscillation (i.e., periodic, low-frequency simulated horizontal/vertical head perturbations) also improves the vection in depth induced by radial flow in a fashion that is remarkably similar to that for this simulated viewpoint jitter, despite marked differences in the expected visual-vestibular conflicts in these two situations (Kim \& Palmisano, 2008; Palmisano, Allison, \& Pekin, 2008; Palmisano, Bonato, Bubka, \& Folder, 2007).

One goal of the present study was to examine the effects that different types of gaze (stationary fixation, directed looking, and gaze shifting) have on these viewpoint jitter and oscillation advantages for vection. In the research outlined above, stationary participants were always free to look wherever they liked while watching the visual selfmotion displays. Recent research by Kim and Palmisano (2008) has shown that adding simulated horizontal viewpoint oscillation $(1 \mathrm{~Hz})$ to radial flow not only enhances the experience of vection in depth induced in physically stationary observers, but also generates specific compensatory eye movements, known as ocular following responses (OFRs; see Miles, Busettini, Masson, \& Yang, 2004). In fact, we found that the OFRs produced when stationary observers viewed horizontally oscillating patterns of radial flow were very similar to the eye movements observed when this display oscillation was generated by the observers' physically moving their heads from side to side. Interestingly, the reported vection experiences were also identical in these two situations. One intriguing explanation of the above null findings is that the OFRs indirectly stimulated the vestibular cortex of our physically stationary observers, thereby decreasing their level of visual-vestibular conflict and increasing the likelihood of compelling vection. In principle, indirect vestibular stimulation could also explain the random viewpoint jitter advantage for vection. However, it would first have to be shown that this jitter generates significant compensatory eye movements, despite its random and high-frequency nature. Thus, in addition to measuring vection, the present study looked for evidence of jitter-induced compensatory eye movements for the first time.

If the above indirect vestibular stimulation account of viewpoint oscillation and jitter effects on vection is valid, incorporating these viewpoint changes into radial flow displays should improve vection only when the stationary observers are free to execute compensatory eye movements. It predicts that these two simulated viewpoint changes will provide little benefit to vection when observers fixate a stationary target superimposed on the self-motion display. However, there is an alternative explanation of the results above, which predicts that stationary fixation should have the opposite effect on vection. It is also possible that the simulated horizontal/vertical viewpoint changes in previous studies improved vection by increasing the retinal slip of the visual displays (de Graaf, Wertheim, \& Bles, 1991; Mach, 1875). According to this explanation, both the jitter and oscillation advantages for vection should be further increased with stationary fixation, since the retinal slip generated by the simulated horizontal/vertical viewpoint changes should increase when compensatory eye movements are suppressed. In partial support of this pro- posal, Tarita-Nistor, Gonzalez, and Ashley (2006) recently showed that the sideways vection induced by (constant velocity) lamellar flow was more compelling when participants fixated a stationary, central target superimposed onto the optic flow, as compared with free-view conditions when no fixation target was present. ${ }^{1}$

A secondary goal of the present study was to reexamine the effect of gaze eccentricity (central vs. peripheral) on vection. There is a widespread belief that vection is independent of gaze eccentricity—based on Post's (1988) finding that circular vection is similar when equal areas of central, mid-peripheral, or far-peripheral vision are stimulated by rotary optic flow. Most subsequent studies supporting eccentricity independence have examined the vection induced by 2-D lamellar or rotary patterns of optic flow, ${ }^{2}$ in which local image velocity remains reasonably constant across the visual field. However, the present experiments examined the effect of gaze eccentricity on vection in depth induced by 3-D jittering, oscillating, or purely radial patterns of expanding optic flow, where the local image velocities always increased with display eccentricity. The effect of gaze eccentricity was tested by presenting fixation targets in desired central/peripheral gaze location(s) (1) before the self-motion display began (directed-looking conditions), (2) both before and during the self-motion display (stationary fixation conditions), or (3) intermittently throughout the self-motion display at various screen locations (gaze-shifting conditions). We predicted that the vection in depth induced by all of our radial flow displays should increase with peripheral, as compared with central, gaze in the present study, for two main reasons: first, because it has been shown that vection generally increases with optic flow speed (Dichgans \& Brandt, 1978), and local image velocities in our radially expanding flow displays always increased toward the periphery; second, because the edges of the nearby, stationary occluding mask - which blocked the rest of the laboratory from view - were directly adjacent to the peripheral gaze location for our optic flow. Similar nearby observerlocked frames of reference have been shown to facilitate vection in the past (Howard \& Heckmann, 1989; Howard \& Howard, 1994). Thus, we predicted that the experience of vection in depth should be more compelling when participants attended to the optically faster peripheral regions of our radial expanding flow displays and to the relative motion between the nearby occluding mask and the more distant peripheral flow.

\section{EXPERIMENT 1A Effects of Gaze and Oscillatory Viewpoint Jitter on Vection}

In this experiment, we examined the effects of gaze type and gaze eccentricity on the vection induced by oscillating and nonoscillating patterns of radial flow. When present, the simulated horizontal/vertical viewpoint oscillation was always low frequency $(1 \mathrm{~Hz})$ and predictable in nature. We have recently shown that this type of oscillating display induces OFRs when viewed freely (Kim \& Palmisano, 2008). In different trials, participants 
either fixated a stationary visible target (OFRs should be suppressed in these conditions) or looked at a previously identified screen location (OFRs would be generated in these conditions) while viewing these two types of self-motion displays. The participants gazed at the center of the self-motion displays for half of the experimental trials and at the periphery of the self-motion displays for the remainder.

\section{Method}

Participants. Seven male and 7 female undergraduate psychology students and staff at the University of Wollongong participated in this experiment (mean age, 26.6 years, $S D=8.5$ years). All had normal or corrected-to-normal vision, were clear of any visual or vestibular impairment, and presented no obvious signs of oculomotor or neurological pathology. A Au\$20 incentive was provided to each participant on completion of the four experimental blocks, which lasted approximately $1 \mathrm{hr}$. The university ethics committee approved the study in advance, and each subject had to provide written informed consent before participating in the study.

Design. Three independent variables were manipulated in this experiment. The first was optic flow type. The visual displays simulated constant-velocity forward self-motion in depth either with or without viewpoint oscillation. When present, viewpoint oscillation was applied along either the observer's horizontal or vertical axis. The second was gaze eccentricity. Prior to the self-motion display, a small, stationary fixation target was presented either in the center or $15^{\circ}$ below the center of the screen. The third was gaze type. In fixation conditions, this fixation target remained visible for the entire $30 \mathrm{sec}$ of the trial, superimposed onto the optic flow. In directedlooking conditions, this fixation target disappeared as soon as the optic flow began. Three dependent variables were measured for each trial: (1) the overall vection strength rating, (2) the vection onset latency, and (3) the total vection duration. These vection measures were similar to those used in previous studies (Palmisano et al., 2008; Palmisano et al., 2007; Palmisano et al., 2003; Palmisano \& Chan, 2004; Palmisano et al., 2000). Trial order was random, with each of the experimental conditions above being tested four times.

Apparatus. Optic flow displays were generated on a Dell Optiplex GX620 PC and front projected onto a $1.48 \mathrm{~m}$ wide $\times 1.20 \mathrm{~m}$ high flat projection screen using a color data projector (Model XD400U, Mitsubishi Electric). The display subtended a visual angle of $43^{\circ} 34^{\prime}$ wide and $32^{\circ} 4^{\prime}$ high when viewed through a large, rectangular viewing tube attached to a head- and chinrest $2 \mathrm{~m}$ distant. The tube blocked the participant's view of his/her stationary surroundings (which included the screen's frame). The onset and total duration of vection were reported by pressing and holding down the trigger of a Logitech Attack 3 joystick. Vection strength ratings were obtained directly following the inducing display by moving the joystick back and forth along the pitch axis. The final strength rating was recorded when a button was pressed on the base of the joystick. The eyetracking equipment will be described in detail in a separate section below.

Visual displays. Prior to each display presentation, a small, stationary green fixation point $\left(0^{\circ} 20^{\prime} ;\right.$ luminance, $\left.8 \mathrm{~cd} / \mathrm{m}^{2}\right)$ was presented for $5 \mathrm{sec}$ either at the center of the screen or at a location $15^{\circ}$ below the center of the display. On half the trials, this fixation point remained visible during the $30-\mathrm{sec}$ self-motion display that followed. On the remainder, it disappeared as soon as the self-motion display began. Each of these self-motion displays consisted of 2,304 randomly positioned blue square objects (luminance, $1.8 \mathrm{~cd} / \mathrm{m}^{2}$ ) on a black background (luminance, $0.03 \mathrm{~cd} / \mathrm{m}^{2}$ ). Objects were distributed uniformly within a simulated 3-D spatial environment ( 8 units wide $\times 8$ units high $\times 18$ units deep). The standard purely radial (i.e., nonoscillating) flow pattern simulated constant-velocity forward self-motion in depth, which was generated by moving the square objects toward the camera viewpoint at a rate of 2.8 units/sec. Once a group of objects had moved beyond the near clipping plane, their spatial configuration was randomized, and they then resumed their movement from the far clipping plane. This ensured the smooth and continuous simulation of self-motion in depth and minimized the processing cost in terms of scene rendering. The size of each square object increased from $0.05^{\circ}$ to $1.08^{\circ}$ with proximity to the camera, which provided the smooth appearance of blue objects flowing from the black background. The oscillating self-motion displays examined in this experiment were identical to the purely radial displays described above, with the sole exception being that they also contained either horizontal or vertical simulated viewpoint oscillation. The combined optic flow was superficially similar to the visual effects of bob or sway produced while walking forward. The amplitude of the viewpoint oscillation was \pm 3 units, and its frequency was held constant at $1 \mathrm{~Hz}$. This translated into peak simulated horizontal or vertical trajectories of head displacement of approximately $\pm 46^{\circ}$.

Procedure. The participants were informed that they would observe displays of moving objects and were told the following:

Sometimes the objects may appear to be moving towards you; at other times you may feel as if you are moving towards the objects. Your task is as follows: If you feel that you are moving forwards, then press the trigger of the joystick and hold it in as long as this experience continues. If you don't feel that you are moving forwards then don't press (or immediately release) the trigger.

The first display presented was the standard stimulus, a purely radial pattern of optic flow with central, stationary fixation. After $10 \mathrm{sec}$ of display exposure had elapsed, the participants were asked whether they felt as if they were moving or stationary. If they responded that they were moving, they were told that the strength of their feeling of self-motion corresponded to a value of 5 (with 0 representing stationary). Following each subsequent optic flow display, an interval scale was presented on the screen $(0-10)$ with a default position of 5 (the modulus). The participants made their vection strength ratings by pulling back or pushing forward on the joystick to position a needle along the bar and pressing a button on the joystick's base to record their vection strength setting. After several practice trials, the experimental trials were presented in a random order; each had a duration of $30 \mathrm{sec}$ and an intertrial interval of $30 \mathrm{sec}$.

Prior to statistical analysis, the vection onsets and total vection durations were determined for each $30-\mathrm{sec}$ trial. Vection onset was measured as the time between the start of the visual self-motion display and the time that the joystick trigger was first pressed. The duration of vection was measured as the total amount of time the trigger of the joystick was held down during the self-motion display. Since there were four replications of each experimental condition, the vection strength, onset, and duration data were averaged before statistical analyses were carried out.

Eyetracking. Eye movement data was obtained via videooculography from 4 of our 14 participants. A dedicated PC and a head-mounted FireWire camera were used to sample $320 \times 240$ pixel images from the left eye under infrared illumination (Figure 1). An infrared opaque mirror was used to angle an image of the eye toward the laterally positioned acquisition camera. This mirror was transparent to natural light, allowing the participant to perform the psychophysical tasks with minimal invasiveness. Each participant initially performed a horizontal and vertical calibration to ascertain the associated changes in pupil positions in pixels that conformed to known angles of deviation between two on-screen fixation targets. Horizontal and vertical eye position data were referenced to the pupil coordinates recorded when the participants fixated on the center of the display. These data were transmitted via a network cable to the local PC used to run the vection experiment, where they were recorded to ASCII files. The root-mean square (RMS) gaze error was computed as a measure of on-screen fixation stability (in degrees). This was essentially the mean standard deviation of the observer's eye position over the period of time that his/her gaze was directed at 


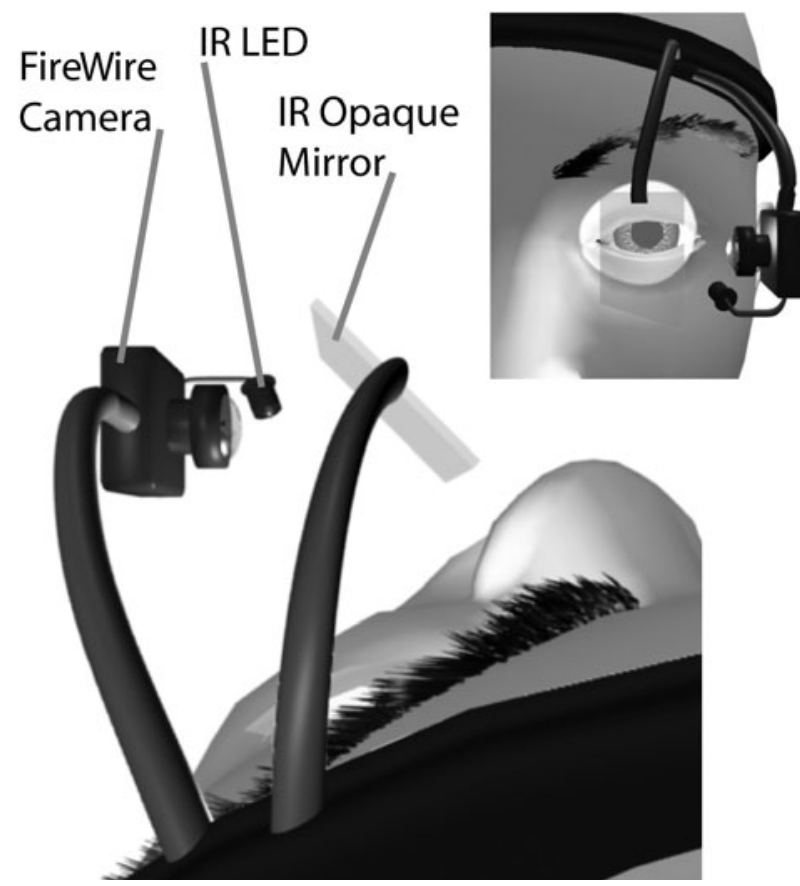

Figure 1. Standard head-mounted apparatus used for video eye movement recording in the present study. IR, infrared; LED, light-emitting diode.

the particular display location. These RMS values were calculated separately for horizontal and vertical eye movements.

\section{Results}

Eyetracking data. Figures 2B and 2A show the vertical eye positions of 1 representative participant (J.B.) while she viewed displays with and without simulated vertical viewpoint oscillation, respectively. The four traces in each figure represent her vertical eye positions during central and peripheral stationary fixation, as well as during central and peripheral directed-looking conditions. Repeated measures $t$ tests were performed on the RMS vertical gaze errors of 4 participants (see also Figure 2D). As was predicted, stationary fixation was found to significantly reduce the gain of the vertical eye movements produced by vertically oscillating patterns of radial flow [as compared with comparable directed-looking conditions; $t(3)=7.48$, $p<.004]$. Stationary fixation was also found to significantly reduce the gain of vertical eye movements produced by purely radial flow during directed-looking conditions $[t(3)=3.42, p<.05]$. In the absence of a fixation target, displays with vertical viewpoint oscillation were found to produce significantly larger vertical eye movements when the participants were directed to look peripherally, as opposed to centrally $[t(3)=3.75, p<.04]$. However, these same peripheral and central directed-looking conditions did not produce significantly different vertical eye movements when the participants were exposed to purely radial flow $[t(3)=0.79, p>.05]$.

Vection strength ratings. A repeated measures ANOVA was performed on the participants' vection strength rating data. Consistent with previous experi- ments, we found a significant main effect of optic flow type on vection strength ratings $[F(2,26)=20.18, p<$ $.0001]$. Bonferroni-corrected post hoc contrasts revealed that (1) oscillating radial flow produced stronger vection ratings than did purely radial flow $(p<.05)$ and $(2)$ horizontal oscillation did not produce vection strength ratings that were significantly different from those for vertical oscillation $(p>.05$; see Figure $3 \mathrm{~A})$. We also found a significant two-way interaction between gaze eccentricity and gaze type on vection strength ratings $[F(1,13)=$ $5.61, p<.03]$. Bonferroni-corrected post hoc contrasts revealed that (1) stable central fixation produced significantly stronger vection ratings than did stable peripheral fixation $(p<.05)$ and $(2)$ central and peripheral looking did not produce significantly different vection ratings $(p>.05$; see Figure 3A). No other main effects or interactions were found to reach significance.

Vection time course. Separate repeated measures ANOVAs were performed on the participants' vection onset and duration data. We found a significant main effect of optic flow type for both vection onsets $[F(2,26)=$ $4.62, p<.02]$ and vection durations $[F(2,26)=8.852, p<$ $.001]$. Bonferroni-corrected post hoc contrasts revealed that (1) oscillating radial flow produced shorter vection onsets and longer vection durations than did purely radial flow (both $p$ s $<.05$ ) and (2) horizontal oscillation did not produce vection onsets or durations significantly different from those for vertical oscillation (both $p \mathrm{~s}>.05$ ). As can be seen in Figures 3B and 3C, we also found a significant three-way interaction in the vection duration data between optic flow type, gaze type, and gaze eccentricity $[F(2,26)=4.04, p<.03]$. Bonferroni-corrected post hoc contrasts revealed that (1) purely radial flow induced significantly longer durations of vection with peripheral, as opposed to central, looking ( $p<.05$ ), (2) purely radial flow did not induce significantly different durations of vection with central and peripheral fixation $(p>.05)$, and (3) oscillating radial flow did not induce significantly different durations of vection with central and peripheral looking $(p>.05)$ or with central and peripheral fixation $(p>.05)$. No other main effects or interactions were found to reach significance.

\section{Discussion}

Contrary to the proposal that simulated viewpoint oscillation should improve vection only when self-motion displays are presented without a superimposed stationary fixation target, we found that $1-\mathrm{Hz}$ display oscillation improved the vection induced in all of the gaze type and eccentricity conditions examined. Adding simulated viewpoint oscillation to radial flow was found to increase vection strength ratings, decrease vection onsets, and increase the total duration of vection. However, contrary to the notion of eccentricity independence, some modest effects of gaze type and gaze eccentricity were observed in the vection data. In the introduction, we predicted that peripheral gaze should induce more compelling vection than central gaze. In support of this notion, we found that peripheral looking did significantly increase the duration of vection induced by purely radial flow (as compared with central-looking conditions). 
A

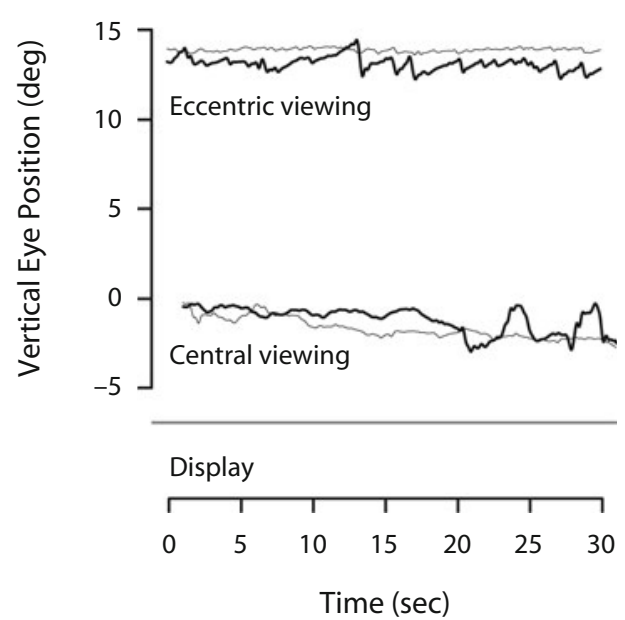

C

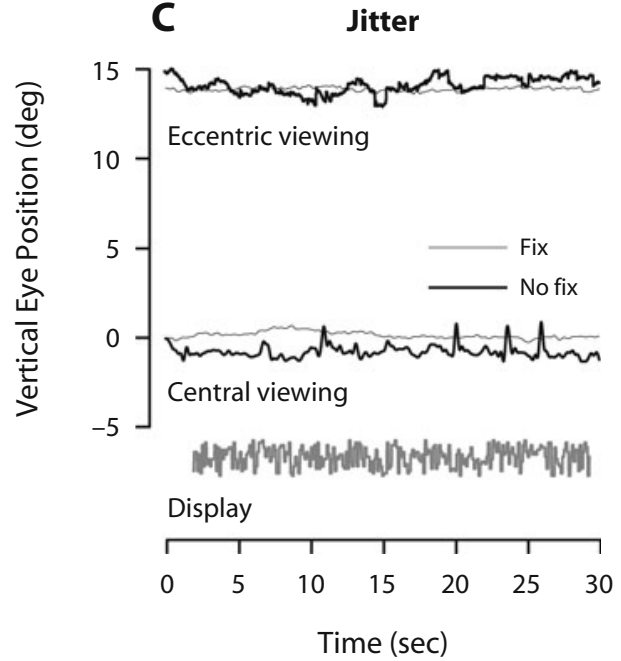

B

Oscillation

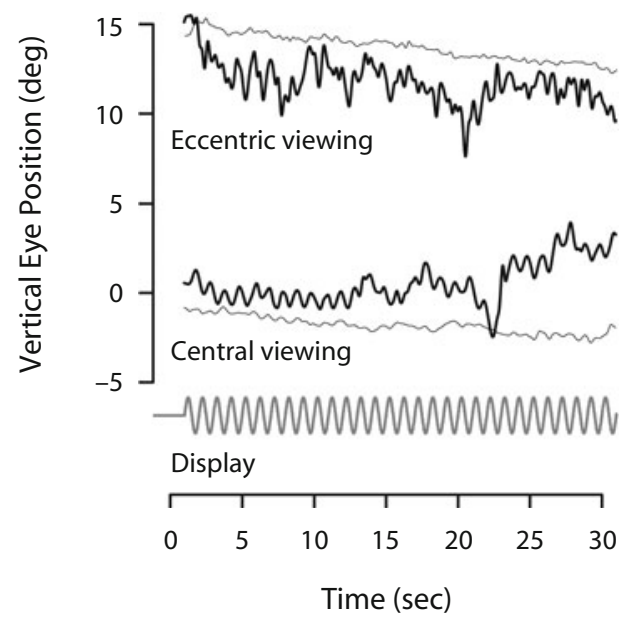

D

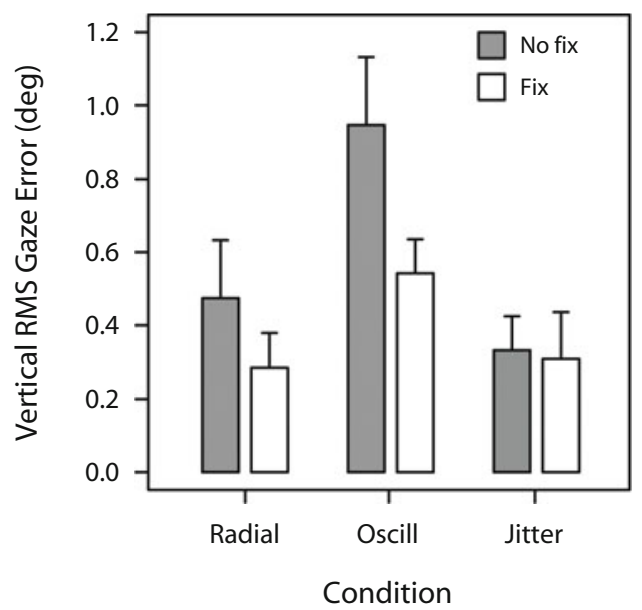

Figure 2. (A-C) Examples of the eye movements made by 1 participant (J.B.) while viewing pure radial flow (A), radial flow with vertical oscillatory viewpoint jitter (B), and radial flow with vertical random viewpoint jitter $(\mathrm{C})$. The lower trace just below each plot shows the amplitude and frequency of the viewpoint oscillation/jitter added to each of these self-motion displays. The light gray traces in each plot show the changes in vertical eye position over time when J.B. fixated a stationary central or peripherally located target while viewing the optic flow. The thick dark traces in each plot show the change in vertical eye position over time when J.B. was directed to look either centrally or peripherally at the optic flow without a visible fixation point. (D) Bar plots show the mean vertical root-mean squared (RMS) gaze errors for 4 of our participants during central and peripheral looking with jittering ("Jitter"), oscillating ("Oscill"), and purely radial ("Radial") patterns of optic flow. Error bars represent standard deviations (Experiments 1A and 1B).

However, no such improvement was observed with oscillating radial flow either for vection duration or for any of the other vection indices measured. In fact, analysis of the vection strength rating data showed that peripheral stationary fixation actually resulted in weaker vection ratings than did central stationary fixation.

One possible explanation for the modest inhibitory effect of peripheral fixation (but not peripheral looking) was that, in these conditions, the stationary fixation target was always visible, perceived to be at a depth similar to that of the optic flow and placed in the region containing the fastest local image velocities. By comparison, when the stationary fixation target was located in the center of the display, its location was close to (in the case of oscillating displays) or coincided with (in the case of nonoscillating displays) the focus of expansion of the radial flow field $^{3}$ (i.e., the point of zero optical velocity in the display). Interestingly, Diels, Ukai, and Howarth (2007) recently used similar stationary fixation conditions and failed to find any significant effect of fixation eccentricity on the vection strength ratings induced by purely radial flow. Although the eccentricities of their peripheral fixation targets were similar to ours $\left(16^{\circ}\right.$ as opposed to $15^{\circ}$ from the center of the display), they used much larger patterns of 

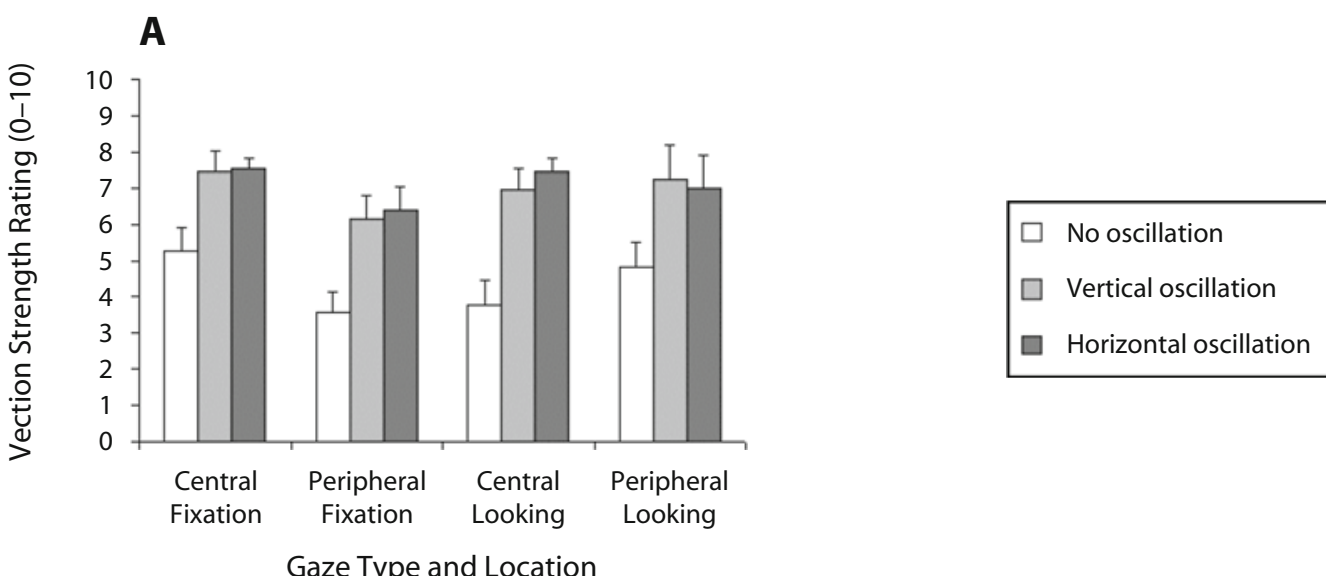

B

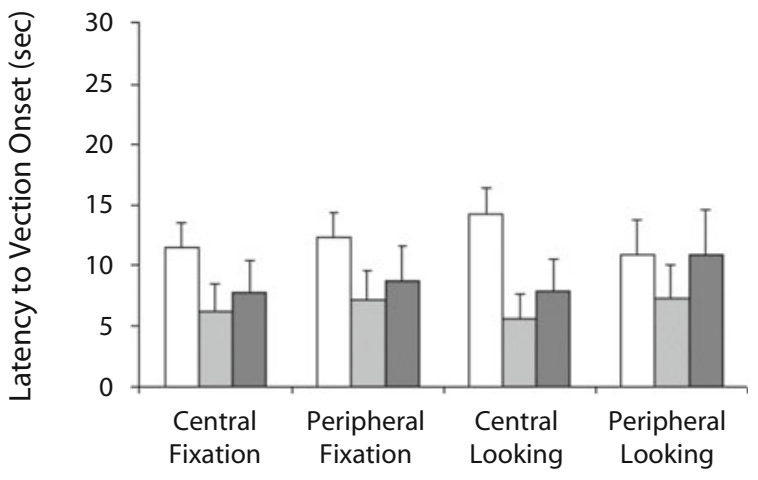

Gaze Type and Location

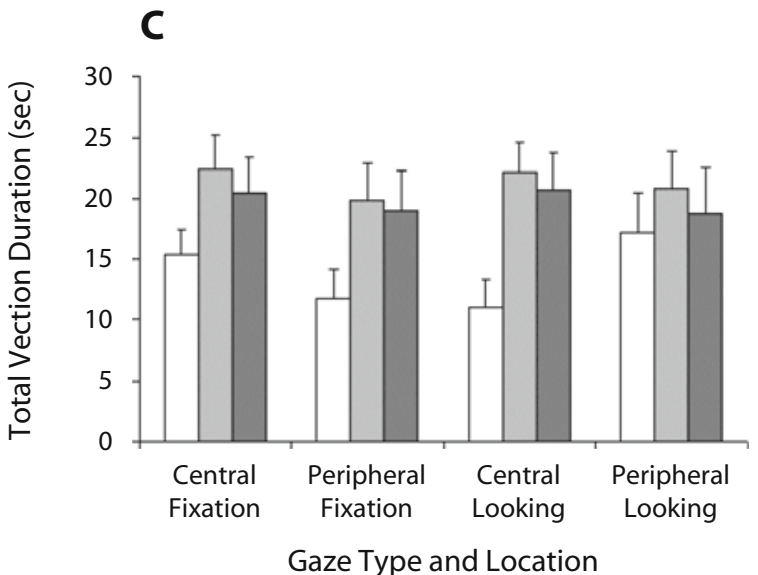

Figure 3. Effects of gaze type (fixation or directed looking) and location (central or peripheral) on the vection induced by self-motion displays either with or without simulated oscillatory viewpoint jitter (i.e., no, vertical, or horizontal oscillation). The vection experience is measured in terms of participant strength ratings $(A)$, latency to vection onset $(B)$, and total vection duration (C). Bar plots represent mean values. Error bars represent the standard errors of the means (Experiment 1A).

optic flow $\left(70^{\circ}\right.$ horizontal $\times 52^{\circ}$ vertical as opposed to $43^{\circ}$ horizontal $\times 32^{\circ}$ vertical in the present study). Because their stationary peripheral fixation targets were located in a region containing only mid-range (as opposed to maximum) image velocities, they might have had less of an inhibitory effect on vection ratings. However, it is also likely that Diels et al.'s failure to find any significant effect of fixation eccentricity on vection strength ratings was due to their extremely long trial durations (each lasting $10 \mathrm{~min}$, as opposed to the $30 \mathrm{sec}$ used in the present study).

\section{EXPERIMENT 1B \\ Effects of Gaze and Random Viewpoint Jitter on Vection}

We have previously shown that simulated viewpoint jitter (random, high frequency ${ }^{4}$ ) can improve the vection induced by radial flow in a very similar fashion to the $1-\mathrm{Hz}$ simulated viewpoint oscillation (periodic, low frequency) examined in Experiment 1A (Palmisano et al., 2008). However, because jittering optic flow is high frequency and random in nature, any compensatory eye movements generated might be very different from those generated by viewpoint oscillation. In Experiment 1B, we examined the effects of gaze type and eccentricity on the vection and compensatory eye movements induced by randomly jittering and nonjittering radial flow.

\section{Method}

Thirteen of the original 14 participants tested in Experiment 1A also completed testing in this follow-up experiment. The apparatus, visual displays, and procedure were identical to those in Experiment $1 \mathrm{~A}$, except that random viewpoint jitter was examined (instead of viewpoint oscillation). Although the amplitude of this random (horizontal/vertical) viewpoint jitter remained the same (ranging from \pm 3 scene units per frame), its frequency could be much higher, capped at $10 \mathrm{~Hz}$. The amplitude of simulated random horizontal/ vertical head displacement ranged within a $\pm 46^{\circ}$ cone at any given instant. Eyetracking was again performed on the same 4 observers that were examined in Experiment 1A.

\section{Results}

Eyetracking data. Figure $2 \mathrm{C}$ shows the vertical eye positions of one representative participant (J.B.) while she viewed displays with and without simulated viewpoint jitter, 


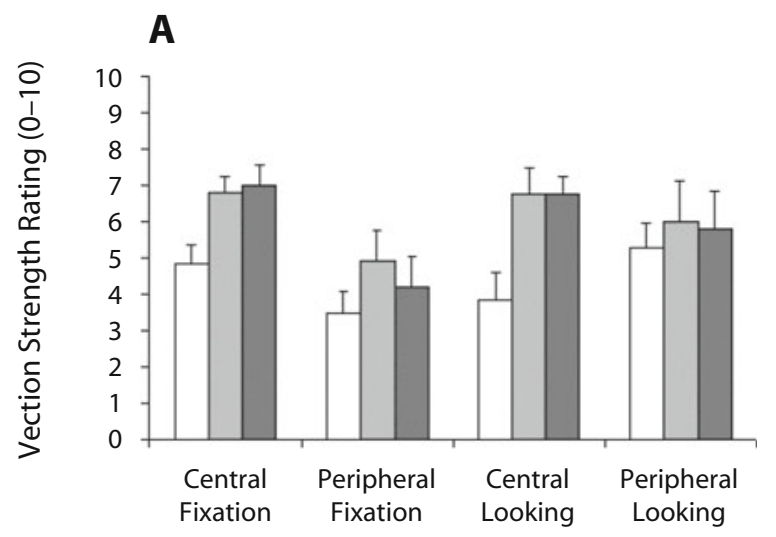

Gaze Type and Location

B

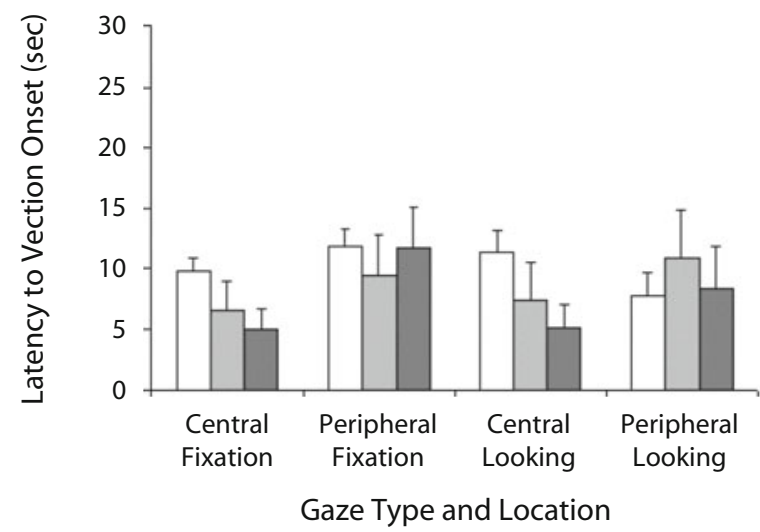

C

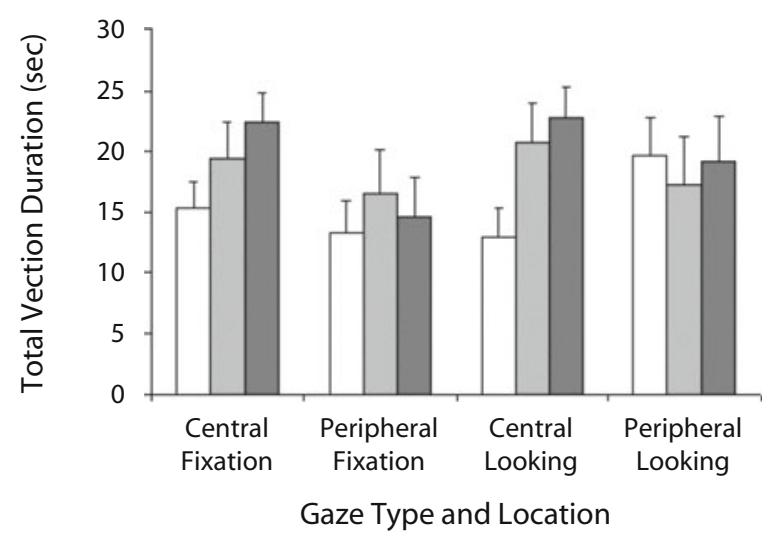

Figure 4. Effects of gaze type (fixation or directed looking) and location (central or peripheral) on the vection induced by self-motion displays either with or without random viewpoint jitter (i.e., no, vertical, or horizontal jitter). The vection experience is measured in terms of participant strength ratings (A), latency to vection onset (B), and total vection duration (C). Bar plots represent mean values. Error bars represent the standard errors of the means (Experiment 1B).

respectively. We performed two repeated measures $t$ tests on the RMS vertical gaze errors of 4 of our participants (see also Figure 2D). It was shown that stationary fixation did not significantly reduce vertical eye movements produced by vertically jittering radial flow [i.e., as compared with directed-looking conditions; $t(3)=1.57, p>$.05]. However, vertical viewpoint jitter was found to produce significantly larger vertical eye movements when the participants were directed to look peripherally, as opposed to centrally $[t(3)=9.41, p<.002]$. The equivalent eyetracking analyses for oscillating and purely radial flow have been provided in the Results section of the previous experiment.

Vection strength ratings. A repeated measures ANOVA was performed on the participants' vection strength rating data. Consistent with previous experiments, we found a significant main effect of optic flow type $[F(2,24)=5.66, p<.01]$. Bonferroni-corrected post hoc contrasts revealed that (1) vection strength ratings were significantly greater for jittering radial flow than for purely radial flow $(p<.05)$ and $(2)$ horizontal display jitter did not produce vection strength ratings that were significantly different from those for vertical display jitter $(p>.05)$. However, as can be seen in Figure 4A, we also found a significant two-way interaction between optic flow type and gaze eccentricity $[F(2,24)=12.87$, $p<.0002]$. Bonferroni-corrected post hoc contrasts revealed that (1) viewpoint jitter increased vection in depth strength ratings more when the participants gazed centrally than when they gazed peripherally $(p<.05)$ and (2) gaze eccentricity did not significantly alter the vection in depth strength ratings for purely radial displays $(p>$ $.05)$. Finally, we found a significant two-way interaction between gaze eccentricity and gaze type $[F(1,12)=8.16$, $p<.01$; see Figure 4A]. Bonferroni-corrected post hoc contrasts revealed that (1) stable central fixation produced significantly stronger vection ratings than did stable peripheral fixation $(p<.05)$ and $(2)$ central and peripheral looking did not produce significantly different vection ratings $(p>.05)$. No other main effects or interactions were found to reach significance.

Vection time course. Separate repeated measures ANOVAs were performed on the participants' vection onset and duration data. We found significant two-way interactions between optic flow type and gaze eccentric- 
ity for both vection onsets $[F(2,24)=4.12, p<.03]$ and vection durations $[F(2,24)=7.77, p<.003$; see Figures $4 \mathrm{~B}$ and $4 \mathrm{C}]$. Bonferroni-corrected post hoc contrasts revealed that (1) viewpoint jitter improved vection more (i.e., shorter onsets and longer durations) when the participants gazed centrally than when they gazed peripherally $(p<.05)$ and $(2)$ gaze eccentricity did not significantly alter the vection time course for purely radial displays $(p>.05)$. We also found significant three-way interactions between optic flow type, gaze type, and gaze eccentricity for vection onsets $[F(2,24)=3.80, p<.04]$ and vection durations $[F(2,24)=3.82, p<.04$; see Figures $4 \mathrm{~B}$ and $4 \mathrm{C}]$. Bonferroni-corrected post hoc contrasts revealed that (1) purely radial displays induced significantly shorter onsets and significantly longer durations of vection with peripheral, as opposed to central, looking $(p<.05)$; (2) purely radial displays did not induce significantly different onsets or durations of vection with central and peripheral fixation $(p>.05)$; (3) jittering displays did not induce significantly different onsets and durations of vection with central and peripheral looking $(p>.05)$; but (4) jittering displays did induce significantly shorter onsets and longer durations of vection with central, as opposed to peripheral, fixation $(p<.05)$. No other main effects or interactions were found to reach significance.

\section{Discussion}

In Experiment 1A, gaze appeared to have little effect on the oscillatory viewpoint jitter advantage for vection. However, in Experiment 1B, significant effects of gaze were observed on the random viewpoint jitter advantage for vection. Viewpoint jitter was shown to produce the greatest vection improvements when the participants looked or fixated centrally. By contrast, the viewpoint jitter advantage for vection was either reduced (in terms of strength ratings) or absent (in terms of the vection time course) when the participants looked or fixated peripherally. The reasons for the reduced effects of viewpoint jitter with peripheral gaze in this experiment appeared to differ depending on whether the participants were looking or fixating.

Peripheral stationary fixation appeared to destroy the viewpoint jitter advantage by impairing the vection induced by both jittering and nonjittering displays. As in Experiment 1A, peripheral stationary fixation was found to produce weaker vection than did central stationary fixation - presumably, because the stationary peripheral fixation target was located in the display region that contained the fastest local image velocities. However, although viewpoint oscillation was still able to improve the vection induced during peripheral fixation in Experiment 1A, viewpoint jitter was unable to produce a similar benefit in the present experiment. Interestingly, of the many experimental manipulations carried out in previous studies, ${ }^{5}$ peripheral stationary fixation is the only one that has been able to destroy this extremely robust vection advantage. It is likely that we failed to find a jitter advantage in this specific situation because the horizontal/vertical image velocities (or the retinal slip) produced by the $10-\mathrm{Hz}$ random jitter in the periphery were so extreme (i.e., much faster than those produced by the $1-\mathrm{Hz}$ viewpoint oscillation in
Experiment 1A) that they exceeded the visual limits for processing self-motion. Thus, according to the retinal slip hypothesis, peripheral fixation destroyed the jitter advantage because, in the absence of compensatory eye movements, the retinal slip generated by the horizontal/vertical jitter was too extreme to be processed.

By contrast, peripheral looking actually appeared to increase the vection induced by purely radial optic flow (above the levels found not only for viewing these displays with central looking, but also as compared with central and peripheral fixation conditions). This finding directly replicates Experiment 1A; as with the original finding, it is again contrary to both the notion of eccentricity independence and the findings of the Diels et al. (2007) study. As was noted earlier, this vection advantage for peripheral looking could be explained by (1) the increasing (but not excessively large) image velocities in the periphery, (2) the proximity of the nearby observer-locked stationary mask, and (3) the lack of any interference from a stationary peripheral fixation target appearing at the same depth as the optic flow. It is possible that the failure to observe a jitter-based vection advantage in these peripheral-looking conditions was due to ceiling effects (i.e., eccentricitybased improvements made it difficult to observe any jitterbased improvements). However, because jitter-induced compensatory eye movements were greater during peripheral looking, the amount of retinal slip produced by jitter in these conditions should have been less than that produced during central looking. This is another reason why the jitter advantage for vection might have been reduced in peripheral-looking conditions.

\section{EXPERIMENT 2 Effects of Gaze Shifting on Vection}

In Experiment 2, we examined the effects of active gaze under less constrained and more realistic viewing conditions (i.e., closer to free-view conditions). When viewing these horizontally/vertically oscillating and purely radial inducing displays, participants were instructed to always look in the location indicated by a briefly flashed fixation cross, which either appeared periodically in the center of the display or alternated its location between the center and periphery of the display.

\section{Method}

The visual displays and procedure were identical to those in Experiment $1 \mathrm{~A}$, with the following exceptions. Five seconds before the start of each self-motion display, a small, stationary green fixation target $\left(0^{\circ} 20^{\prime} ;\right.$ luminance, $\left.8 \mathrm{~cd} / \mathrm{m}^{2}\right)$ was displayed in the center of the screen. This fixation target disappeared as soon as the self-motion display began. In stable gaze conditions, it was replaced by a large fixation cross $\left(4^{\circ}\right.$; luminance, $\left.8 \mathrm{~cd} / \mathrm{m}^{2}\right)$, which appeared for only $250 \mathrm{msec}$ every $4 \mathrm{sec}$ and, when visible, was always located directly in the center of the display. Gaze-shifting conditions were identical, except that the large fixation cross could be flashed at five different display locations (the center of the screen or $15^{\circ}$ to the left, to the right, below, or above this location). In these gaze-shifting conditions, the fixation cross presentation order was always the same: above first, center next, then left, center, right, center, below, and finally back to the center. The participants were told to direct their gaze toward the location identified by the flashed green cross and to 
keep looking in this direction until the cross reappeared in another/ same location, at which time they should reposition their gaze, if necessary. In the case of gaze shifting, it was hoped that the highly predictable changes in cross location would help the participants quickly and efficiently reposition their gaze.

Participants. Five male and 7 female staff and students at the University of Wollongong participated in this experiment (mean age, 32 years; $S D=4.4$ years). Only two of these had previously participated in Experiments 1A and 1B. All had normal or corrected-to-normal vision, were clear of any visual or vestibular impairment, and presented no obvious signs of oculomotor or neurological pathology. A Au\$20 incentive was provided to each participant on completion of the two experimental blocks, which lasted in total approximately $45 \mathrm{~min}$ (including a 5-min break). The university ethics committee approved the study in advance, and each participant provided written informed consent before participating in the study.

Design. Two independent variables were manipulated in this experiment. The first was optic flow type. Visual displays simulated constant-velocity forward self-motion either with or without horizontal/vertical viewpoint oscillation. The second was gaze type. The participants either maintained a stable central gaze (stable gaze) or alternated their gaze between the center and the periphery of the display (gaze shifting). Three dependent variables were again measured for each trial: (1) the overall vection strength rating, (2) the vection onset latency, and (3) the total vection duration. Trial order was random, with each of the above experimental conditions being tested four times. Eyetracking was again performed in real time via video-oculography on 5 of our 13 participants.

\section{Results}

Eyetracking data. Eye movement data from 5 of our experimental participants are provided in Figure 5. These eye movement recordings confirmed that gaze was directed toward and maintained at the approximate location of the briefly flashed on-screen fixation targets (see Figure 5A). However, examination of the average RMS error in gaze position showed that gaze was significantly more variable during gaze-shifting conditions (i.e., less stabilized on the centrally/peripherally indicated target) than during stable gaze conditions (see Figure 5B). This difference was found to be significant by repeated measures $t$ tests on the averaged data in the horizontal $[t(4)=$ $7.91, p<.005]$ and vertical $[t(4)=4.37, p<.05]$ gaze directions. As can be seen from the lower figure, horizontal instability was greater following a horizontal shift in gaze (e.g., from the center to the left of the display), whereas vertical instability was greater following a vertical shift in gaze (e.g., from the center to the bottom of the display). This was also supported by repeated measures $t$ tests performed on the different eccentricities of gaze in the horizontal $[t(4)=15.73, p<.0001]$ and vertical $[t(4)=6.60, p<.005]$ directions.

Vection strength ratings. A repeated measures ANOVA was performed on the vection strength rating data. As in previous studies, we found a significant main effect of optic flow type on vection strength ratings $[F(2,22)=$ $22.04, p<.0001]$. Bonferroni-corrected post hoc contrasts revealed that (1) oscillating radial flow produced stronger vection ratings than did purely radial flow $(p<$ .05 ) and (2) there was no significant difference between the strength ratings for displays with horizontal and vertical viewpoint oscillation $(p>.05)$. We also found a main

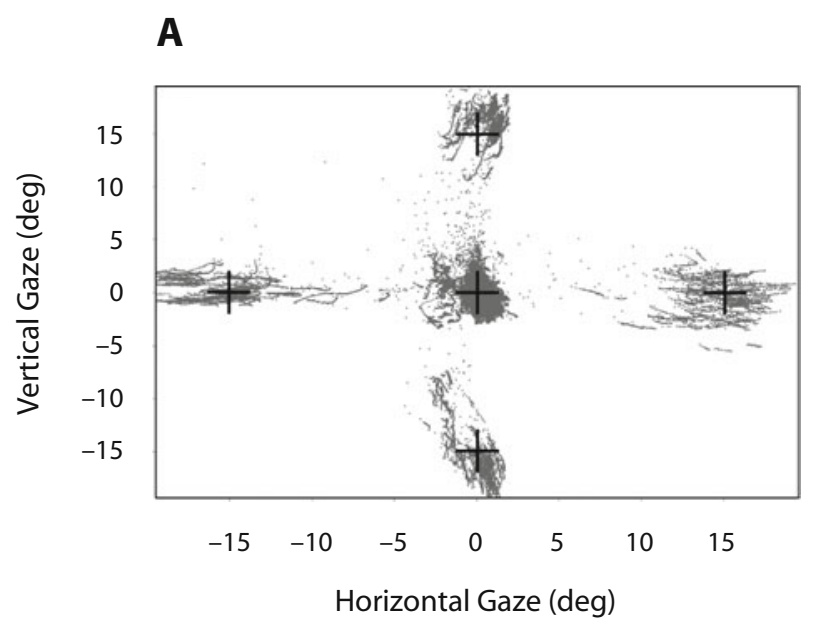

B

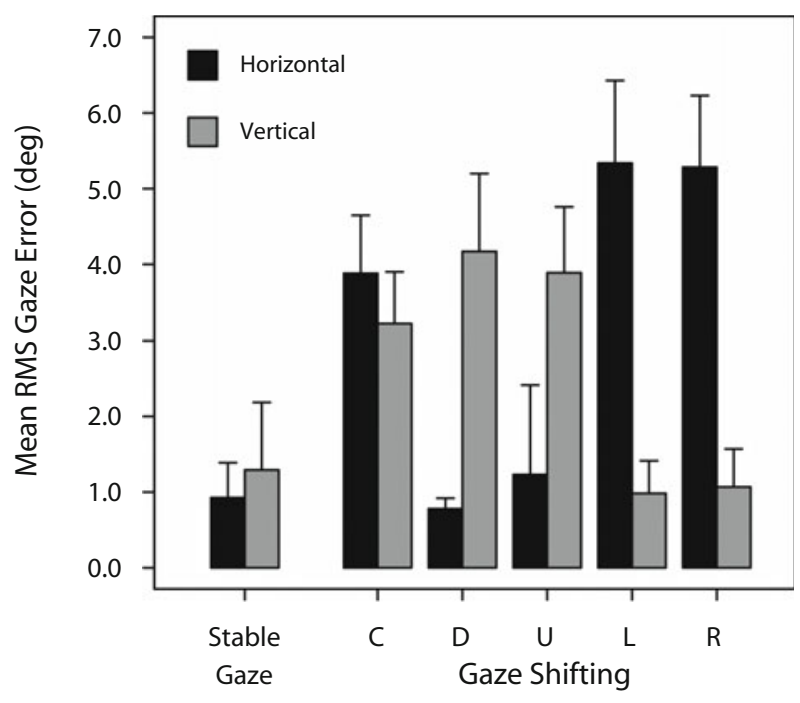

Figure 5. (A) Plots showing a summary of the horizontal and vertical gaze positions during the various phases of a gaze-shifting trial (relative to the locations of the briefly flashed on-screen fixation targets [crosses]). (B) Bar plots representing the average root-mean squared (RMS) error of horizontal and vertical gaze position for 5 participants during stable central gaze and when gaze shifted between central $(C)$, downward (D), upward (U), leftward $(L)$, and rightward $(R)$ targets. Error bars represent standard deviations (Experiment 2).

effect of gaze type $[F(1,11)=23.80, p<.0005]$ and an interaction between gaze type and optic flow $[F(2,22)=$ $5.41, p<.01]$. Bonferroni-corrected post hoc contrasts revealed that gaze shifting produced significantly stronger vection ratings than did stable central gaze for horizontally oscillating, vertically oscillating, and purely radial flow ( $p<.05$ in all three cases). However, as we can see from Figure 6A, gaze shifting improved the vection strength ratings more for purely radial flow than for vertically oscillating and horizontally oscillating radial flow.

Vection time course. Separate repeated measures ANOVAs were performed on the vection onset and dura- 


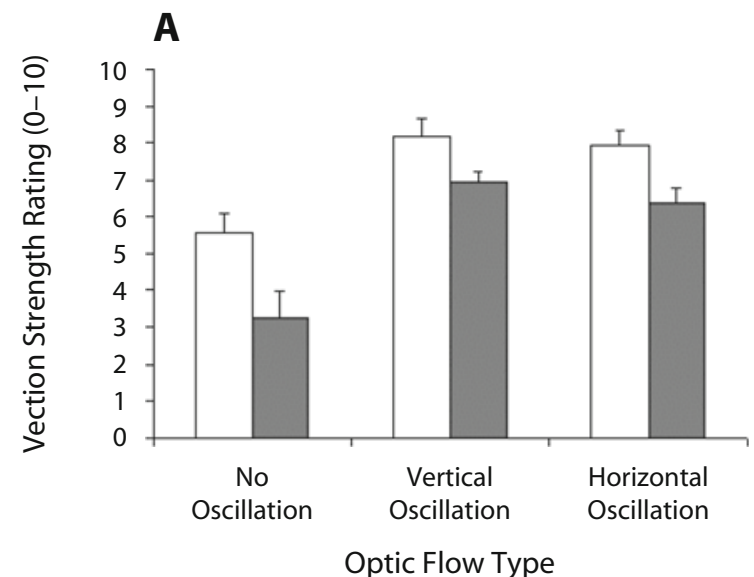

Gaze shifting

Gaze stationary

B

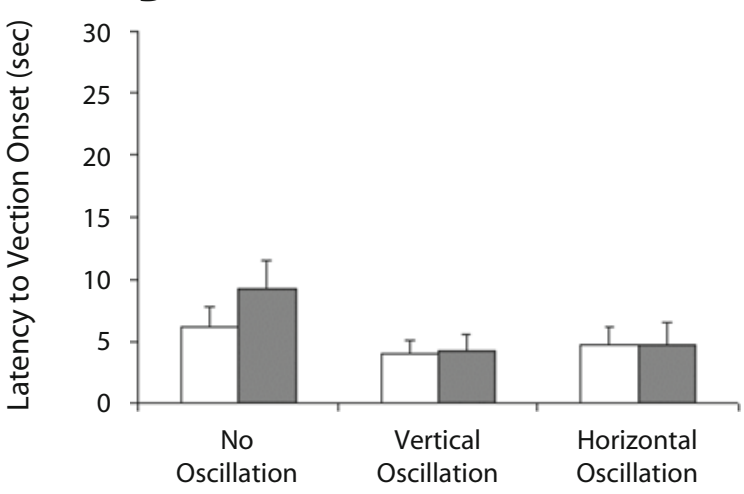

Optic Flow Type

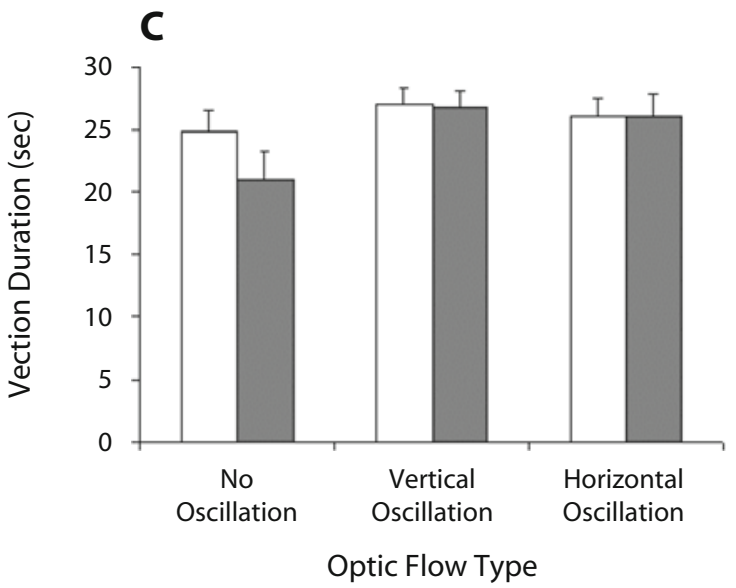

Figure 6. Effect of gaze type (gaze shifting and gaze stationary) on the vection induced by self-motion displays either with or without oscillatory viewpoint jitter (i.e., no, vertical, or horizontal oscillation). The vection experience is measured in terms of participant strength ratings (A), latency to vection onset (B), and total vection duration (C). Bar plots represent mean values. Error bars represent the standard errors of the means (Experiment 2).

tion data. We found a significant main effect of optic flow type on both vection onsets $[F(2,22)=7.54, p<.003]$ and durations $[F(2,22)=8.57, p<.002]$. Bonferronicorrected post hoc contrasts revealed that (1) oscillating radial flow produced shorter vection onsets and longer vection durations than did purely radial flow $(p<.05)$ and (2) there were no significant differences between the vection onsets and durations for displays with horizontal and vertical viewpoint oscillation $(p>.05)$. We also found significant main effects of gaze type [vection onsets, $F(1,11)=6.42, p<.02$; vection durations, $F(1,11)=$ $8.67, p<.01]$ and significant two-way interactions between optic flow type and gaze type for both time course measures [vection onsets, $F(2,22)=6.98, p<.005$; vection durations, $F(2,22)=10.41, p<.0007]$. As can be seen from Figures $6 \mathrm{~B}$ and $6 \mathrm{C}$, the results clearly show that gaze shifting did not significantly alter the vection onsets or durations when displays contained viewpoint oscillation. However, gaze shifting did significantly improve the vection onsets and durations of displays without viewpoint oscillation.

\section{Discussion}

As in Experiment 1A and previous studies, we found that simulated horizontal/vertical viewpoint oscillation significantly improved the vection induced by radial patterns of optic flow. This was true for both gaze-shifting and stable central gaze conditions. Overall, we found that gaze-shifting conditions tended to induce more compelling vection than did stable central gaze conditions. However, this finding was driven primarily by the effect of gaze shifting on the vection induced by purely radial optic flow. Gaze shifting significantly improved both the strength and the time course of the vection induced by displays without viewpoint oscillation (relative to stable, central gaze). Gaze shifting also significantly improved the strength of the vection induced by displays with viewpoint oscillation but had little effect on the time course of vection in this case.

\section{GENERAL DISCUSSION}

In the present study, we examined the effects of stationary fixation, directed looking, and gaze shifting on the 
vection induced by jittering, oscillating, and purely radial patterns of optic flow. Contrary to the notion of eccentricity independence for vection, we found that peripheral stationary fixation impaired the vection, whereas peripheral directed looking improved the vection induced by radial flow (as compared with central fixation and directed-looking conditions). We also discovered a novel gaze-shifting advantage for vection in depth, where shifting gaze from the center to the periphery of the radial display tended to increase/improve vection (as compared with stable central gaze conditions). This gaze-shifting advantage was more pronounced in the vection induced by purely radial flow but was still evident when radial flow displays also simulated horizontal/vertical viewpoint changes. As in the recent study by Palmisano et al. (2008), it was shown that simulated viewpoint oscillation (periodic, $1 \mathrm{~Hz}$ ) improves the vection induced by radial flow in a fashion very similar to that for simulated viewpoint jitter (random, capped at $10 \mathrm{~Hz}$ ). However, although both viewpoint oscillation and jitter were found to produce similar vection advantages during central fixation and central looking (as can be seen in Figures 3 and 4), the former vection advantage proved to be far more robust when gaze eccentricity increased. In fact, peripheral stationary fixation was actually found to destroy the random viewpoint jitter advantage for vection.

In the introduction, it was proposed that stationary fixation might prevent the viewpoint jitter and oscillation advantages for vection by suppressing optokinetic/ compensatory eye movements. Our eyetracking data confirmed that stationary fixation did significantly reduce the OFRs produced by horizontally/vertically oscillating radial flow, as compared with directed-looking conditions (see Figures 2A and 2B for typical eye movements from a representative participant, J.B.). However, contrary to the notion that the viewpoint oscillation advantage for vection would be destroyed by this OFR suppression, oscillating radial flow was still found to induce more compelling vection than purely radial flow when observers maintained (either central or peripheral) stationary fixation. Unlike the OFRs produced by oscillating radial flow, the eye movements produced by jittering radial flow were not found to be significantly different during stationary fixation and directedlooking conditions (see Figure 2C). Thus, eye movement suppression can also not be used to explain the finding that the viewpoint jitter advantage for vection was destroyed by peripheral (but not central) stationary fixation.

The most likely explanation for why peripheral fixation always impaired vection in the present experiments was that these conditions provided a stationary fixation target, which was perceived to be located at the same depth as the optic flow and was placed in the region containing the fastest visual motion. This was true for randomly jittering, oscillating, and purely radial patterns of optic flow alike. The result was that this stationary peripheral fixation target not only impaired the vection induced by purely radial flow, but also contributed to our failure to find a random viewpoint jitter advantage for vection. During central fixation, however, the stationary fixation target either was close to or coincided with the location of the focus of expansion of the optic flow. Since the local image velocities would have been modest in this region, little or no vection impairment would have been expected in this case. Support for this explanation is provided by findings that central fixation did not impair the vection induced by purely radial flow and did not destroy the random viewpoint jitter advantage for vection.

Importantly, Experiment 2 showed that alternating one's gaze between the center and the periphery of the visual self-motion display produced marked improvements in vection (as compared with conditions in which the participant maintained a stable central gaze). Although gaze shifting was shown to improve the vection induced by both oscillating and purely radial patterns of optic flow, the benefits were greater in the case of the latter. To our knowledge, this gaze-shifting advantage for vection in depth has not been reported in any previous study. It is likely that this advantage was due to the increased retinal slip produced when the participants made saccades from the center to the periphery of the display. Eleven of our 12 participants spontaneously reported a simulated self-acceleration when they shifted their gaze from the center of the display to the periphery. There was apparently little change in perceived speed when gaze was shifted back from the periphery to the center of the display. This report also appears to be consistent with the findings of Experiments 1A and 1B that peripheral directed looking actually increased the vection induced by purely radial flow displays, as compared with central directed looking. Retinal slip can also be used to explain why the gaze-shifting advantage was greater for purely radial displays, since displays with viewpoint oscillation would have already contained some degree of retinal slip even when the participants attempted to hold their gaze stable at the center of the display.

Taken together, the results of all three experiments are generally consistent with the notion that vection in depth increases with the degree of retinal slip. As was noted above, the comparison of stationary fixation and directed looking did not appear to be a fair test of this retinal slip hypothesis, since the presence of a stationary fixation target superimposed onto the display was often found to have unexpected inhibitory effects on vection (even when it had little or no impact on participants' eye movements). However, if one considers the results from only the directedlooking conditions, it is clear that conditions that should have produced greater retinal slip also produced more compelling vection. For example, peripheral directed looking at purely radial patterns of optic flow should have produced more retinal slip than should central directed looking and was, in fact, also found to produce significantly more compelling vection. The finding that peripheral (as opposed to central) directed looking did not provide any further vection improvement for oscillating patterns of radial flow can be simply explained by the vection's already being at ceiling levels (due to the increased retinal slip generated by this horizontal/vertical oscillation alone). In a similar fashion, we can also explain the large gaze-shifting vection advantage for purely radial flow and the modest gaze-shifting vection advantage for oscillating radial flow, based on retinal slip. However, from the present findings, it seems likely that vection increases with retinal slip only up to a point. Most psychophysical evidence suggests that 
the visual system prefers self-motion information based on low temporal frequencies (Berthoz et al., 1975; Previc, Kenyon, Boer, \& Johnson, 1993). Although local image velocities and retinal slip should have increased with the gaze eccentricity for all three display types (jittering, oscillating, and purely radial flow), the increase would have been far greater with displays containing random viewpoint jitter. We conclude that the extremely fast peripheral motion, and resulting retinal slip, produced by this random viewpoint jitter (capped at $10 \mathrm{~Hz}$ ) exceeded the limits of visual self-perception.

Contrary to the notion of eccentricity independence for vection and the recent null findings of Diels et al. (2007), we conclude that where and how one looks at a visual selfmotion display can significantly alter the overall vection experience. We have shown that peripheral looking and gaze shifting can significantly improve the vection produced by displays simulating constant-velocity self-motions or lowfrequency viewpoint oscillation, by exposing the observer to faster local image velocities, nearby observer-fixed references, and increased retinal slip. However, it appears that central gaze might be required for compelling vection when an observer is exposed to self-motion displays with highfrequency optic flow. The latter finding appears to reflect the finite processing limits of visual self-motion perception.

\section{AUTHOR NOTE}

We thank Luke Downie for running the pilot experiments related to this study. This research was supported by Australian Research Council Discovery Grant DP0772398. Correspondence should be addressed to S. Palmisano, School of Psychology, University of Wollongong, Wollongong, NSW 2522, Australia (e-mail: stephenp@uow.edu.au).

\section{REFERENCES}

Berthoz, A., Pavard, B., \& Young, L. R. (1975). Perception of linear horizontal self-motion induced by peripheral vision (linear vection). Experimental Brain Research, 23, 471-489.

de Graaf, B., Wertheim, A. H., \& Bles, W. (1991). The AubertFleischl paradox does appear in visually induced self-motion. Vision Research, 31, 845-849.

DichGans, J., \& BRANDT, T. (1978). Visual-vestibular interaction: Effects on self-motion perception and postural control. In R. Held, H. Leibowitz, \& H. L. Teuber (Eds.), Handbook of sensory physiology: Vol. 8. Perception (pp. 756-804). New York: Springer.

Diels, C., UKaI, K., \& HowarTh, P. A. (2007). Visually induced motion sickness with radial displays: Effects of gaze angle and fixation. Aviation, Space, \& Environmental Medicine, 78, 659-665.

GiBson, J. J. (1966). The senses considered as perceptual systems. Boston: Houghton Mifflin.

HowARD, I. P. (1982). Human visual orientation. Chichester, U.K.: Wiley.

HowARD, I. P., \& HeCKMANN, T. (1989). Circular vection as a function of relative sizes, distances, and positions of two competing displays. Perception, 18, 657-665.

Howard, I. P., \& Howard, A. (1994). Vection: The contributions of absolute and relative visual motion. Perception, 23, 745-751.

Johansson, G. (1977). Studies on the visual perception of locomotion. Perception, 6, 365-376.

Kim, J., \& PaLmisano, S. (2008). Effects of active and passive viewpoint jitter on vection in depth. Brain Research Bulletin, 77, 335-342.

Lishman, J. R., \& LeE, D. N. (1973). The autonomy of visual kinaesthesis. Perception, 2, 287-294.

MACH, E. (1875). Grundlinien der Lehre von den Bewegungsempfindungen. Leipzig: Engelmann.

Melcher, G. A., \& Henn, V. (1981). The latency of circular vection during different accelerations of the optokinetic stimulus. Perception \& Psychophysics, 30, 552-556.
Miles, F. A., Busettini, C., Masson, G. S., \& Yang, D. S. (2004). Short-latency eye movements: Evidence for rapid, parallel processing of optic flow. In L. M. Vaina, S. A. Beardsley, \& S. K. Rushton (Eds.), Optic flow and beyond (pp. 78-107). Dordrecht: Kluwer.

OMAN, C. M. (1982). A heuristic mathematical model for the dynamics of sensory conflict and motion sickness. Acta Oto-Laryngologica, 44(Suppl. 392), 1-44.

Palmisano, S., Allison, R. S., \& Pekin, F. (2008). Accelerating selfmotion displays produce more compelling vection in depth. Perception, 37, 22-33.

Palmisano, S., Bonato, F., BubKa, A., \& Folder, J. (2007). Vertical display oscillation increases vection in depth and simulator sickness. Aviation, Space, \& Environmental Medicine, 78, 951-956.

Palmisano, S., Burke, D., \& Allison, R. S. (2003). Global perspective jitter induces visual illusions of self-motion. Perception, 32, 97-110.

Palmisano, S., \& Chan, A. Y. C. (2004). Jitter and size effects on vection are immune to experimental instructions and demands. Perception, 33, 987-1000.

Palmisano, S., \& Gillam, B. [J.] (1998). Stimulus eccentricity and spatial frequency interact to determine circular vection. Perception, 27, 1067-1077.

Palmisano, S., Gillam, B. J., \& Blackburn, S. G. (2000). Global perspective jitter improves vection in central vision. Perception, 29, 57-67.

Post, R. B. (1988). Circular vection is independent of stimulus eccentricity. Perception, 17, 737-744.

Previc, F. H., Kenyon, R. V., Boer, E. R., \& Johnson, B. H. (1993). The effects of background visual roll stimulation on postural and manual control and self-motion perception. Perception \& Psychophysics, 54, 93-107.

REAson, J. (1978). Motion sickness adaptation: A neural mismatch model. Journal of the Royal Society of Medicine, 71, 819-829.

Reason, J., \& BRAND, J. (1975). Motion sickness. London: Academic Press.

Tarita-Nistor, L., GonZalez, E. G., \& Ashley, J. (2006). Linear vection as a function of stimulus eccentricity, visual angle, and fixation. Journal of Vestibular Research, 16, 265-272.

TeiXeira, R. A., \& Lackner, J. R. (1979). Optokinetic motion sickness: Attenuation of visually-induced apparent self-rotation by passive head movements. Aviation, Space, \& Environmental Medicine, 50, 264-266.

Wong, S. C. P., \& Frost, B. J. (1981). The effect of visual-vestibular conflict on the latency of steady-state visually induced subjective rotation. Perception \& Psychophysics, 30, 228-236.

Young, L. R., Dichgans, J., Murphy, R., \& BRandT, T. (1973). Interaction of optokinetic and vestibular stimuli in motion perception. Acta Otolaryngologica, 76, 24-31.

ZACHARIAS, G. L., \& YounG, L. R. (1981). Influence of combined visual and vestibular cues on human perception and control of horizontal rotation. Experimental Brain Research, 41, 159-171.

\section{NOTES}

1. It should be noted that the large-amplitude eye movements (optokinetic nystagmus) produced by their lamellar flow are very different from the modest eye movements produced by the radial flow used in the present study (see Miles et al., 2004).

2 . That is, simulating purely sideways linear self-motion or yaw selfrotation. It should be noted that Palmisano and Gillam (1998) have shown that eccentricity independence fails even with these types of displays.

3 . The location of the central fixation point coincided only with the average location of the focus of expansion of the jittering displays, since this focus jittered horizontally or vertically around the center of the display from one instant to the next.

4. For example, capped at $30 \mathrm{~Hz}$.

5. In free-view conditions, the random viewpoint jitter advantage for vection has been shown to persist with jitter frequencies ranging from 1 to $30 \mathrm{~Hz}$ and with jitter amplitudes ranging from one fourth to equal that of the radial flow component.

(Manuscript received March 9, 2009; revision accepted for publication July 7, 2009.) 